\title{
Numerical Solutions of Second Order Initial Value Problems of Bratu-Type via Optimal Homotopy Asymptotic Method
}

\author{
Mohamed Abdalla Darwish, Bothayna S. Kashkari \\ Department of Mathematics, Sciences Faculty for Girls, King Abdulaziz University, Jeddah, KSA \\ Email: dr.madarwish@gmail.com, bkashkari@kau.edu.sa
}

Received 26 December 2013; revised 26 January 2014; accepted 1 February 2014

Copyright $@ 2014$ by authors and Scientific Research Publishing Inc.

This work is licensed under the Creative Commons Attribution International License (CC BY). http://creativecommons.org/licenses/by/4.0/

c) (i) Open Access

\begin{abstract}
We present the optimal homotopy asymptotic method (OHAM) to find the numerical solution of the second order initial value problems of Bratu-type. We solve some examples to illustrate the validity and efficiency of the method.
\end{abstract}

\section{Keywords}

Initial-Value Problem; Bratu; Numerical Solution; Optimal Homotopy Asymptotic Method

\section{Introduction}

Herişanu et al. [1] proposed a new technique called the optimal homotopy asymptotic method (OHAM). The main advantage of OHAM is that it is reliable and straight forward. Also, the OHAM does not need to worry about $h$ curves as homotopy asymptotic method (HAM). Moreover, the OHAM provides controls the convergence of the series solution and its solution agrees with the exact one at large domains, for more information see [2]-[6].

On the other hand, the standard Bratu problem is used in a large variety of applications, such as the fuel ignition model of the theory of thermal combustion, the thermal reaction process model, the Chandrasekhar model of the expansion of the universe, radiative heat transfer, nanotechnology and theory of chemical reaction, for more information see [7] [8] and references therein.

The Bratu initial value problems have been studied extensively because of its mathematical and physical properties. In [9], Batiha studied a numerical solution of Bratu-type equations by the variational iteration method; Feng et al. [10] considered Bratu's problems by means of modified homotopy perturbation method; Rashidinia et al. [11] applied Sinc-Galerkin method for numerical solution of the Bratu's problems; Syam and Hamdan [12] 
used variational iteration method for numerical solutions of the Bratu-type problems; Wazwaz [13] applied Adomian decomposition method to study the Bratu-type equations.

The main goal of this paper is to extend OHAM method to solve the initial value problems of second order differential equations of Bratu-type. The OHAM is very useful to get an approximate solution of the initial value problems of second order differential equations of Bratu-type. Our numerical examples of OHAM are compared with exact ones.

\section{Analysis of OHAM}

In this section we start by describing the basic formulation of OHAM, see for example [1] [3]-[5]. Consider the boundary value problem

$$
\left\{\begin{array}{l}
L(u(x))+g(x)+N(u(x))=0 \\
B\left(u, \frac{\mathrm{d} u}{\mathrm{~d} x}\right)=0
\end{array}\right.
$$

where $g=g(x)$ is a given function and $u=u(x)$ is an unknown function. Here, $L, N$ and $B$ represent a linear operator, a nonlinear operator and a boundary operator, respectively.

By means of OHAM one constructs a homotopy $h(x, p): \mathbb{R} \times[0,1] \rightarrow \mathbb{R}$, which satisfies the following family of equations

$$
\left\{\begin{array}{l}
(1-p)[L(h(x, p))+g(x)]=H(p)[L(h(x, p))+g(x) N(h(x, p))] \\
B\left(h(x, p), \frac{\partial h(x, p)}{\partial x}\right)=0,
\end{array}\right.
$$

where $p \in[0,1]$ is an embedding parameter, $H(p)$ is a non-zero auxiliary function for $p \neq 0$ and $H(0)=0$. It is easy to see that when $p=0$ and $p=1$ we have $h(x, 0)=u_{0}(x)$ and $h(x, 1)=u(x)$, respectively, where $u_{0}(x)$ is obtained from (2.2) for $p=0$

$$
\left\{\begin{array}{l}
L\left(u_{0}(x)\right)+g(x)=0, \\
B\left(u_{0}(x), 0\right)=0 .
\end{array}\right.
$$

Therefore, the unknown function $h(x, p)$ goes from $u_{0}(x)$ to $u(x)$ as $p$ changes from 0 to 1 . In the sequel, we choose auxiliary function $H(p)$ in the form

$$
H(p)=c_{1} p+c_{2} p^{2}+c_{3} p^{3}+\cdots,
$$

where $c_{i}, \quad i=1,2,3, \cdots$, are constants to be determined.

In order to obtain an approximate solution, we expand $h\left(x, p, c_{i}\right), i=1,2,3, \cdots$, in the form of Taylor's series about $p$ as

$$
h\left(x, p, c_{i}\right)=u_{0}(x)+\sum_{j=1}^{\infty} u_{j}\left(x, c_{i}\right) p^{j}, i=1,2,3, \cdots .
$$

Now, substituting by Equation (2.5) into Equation (2.2) and equating the coefficients of like powers of $p$ in the resulting equation, we obtain the governing problem of $u_{0}(x)$, given by Equation (2.3). In addition, the governing problems of $u_{1}(x)$ and $u_{2}(x)$ are given in the forms

and

$$
\left\{\begin{array}{l}
L\left(u_{1}(x)\right)+g(x)=c_{1} N_{0}\left(u_{0}(x)\right), \\
B\left(u_{1}(x), \frac{\mathrm{d}}{\mathrm{d} x} u_{1}(x)\right)=0
\end{array}\right.
$$

$$
\left\{\begin{array}{l}
L\left(u_{2}(x)\right)=L\left(u_{1}(x)\right)+c_{2} N_{0}\left(u_{0}(x)\right)+c_{1}\left[L\left(u_{1}(x)\right)+N_{1}\left(u_{0}(x), u_{1}(x)\right)\right], \\
B\left(u_{2}(x), \frac{\mathrm{d}}{\mathrm{d} x} u_{2}(x)\right)=0,
\end{array}\right.
$$


respectively. Also, the general governing problems of $u_{j}(x)$ are given by

$$
\left\{\begin{aligned}
& L\left(u_{j}(x)\right)= L\left(u_{j-1}(x)\right)+c_{j} N_{0}\left(u_{0}(x)\right) \\
&+\sum_{i=1}^{j-1} c_{i}\left[L\left(u_{j-i}(x)\right)+N_{j-i}\left(u_{0}(x), u_{1}(x), \cdots, u_{j-1}(x)\right)\right] \\
& B\left(u_{j}(x), \frac{\mathrm{d}}{\mathrm{d} x} u_{j}(x)\right)=0, j=2,3,4, \cdots,
\end{aligned}\right.
$$

where $N_{m}\left(u_{0}(x), u_{1}(x), \cdots, u_{j-1}(x)\right)$ is the coefficient of $p^{m}$ in the expansion of $N(h(x, p))$ about the embedding parameter $p$ :

$$
N\left(h\left(x, p, c_{i}\right)\right)=N_{0}\left(u_{0}(x)\right)+\sum_{m=1}^{\infty} N_{m}\left(u_{0}, u_{1}, \cdots, u_{m}\right) p^{m},
$$

where $h\left(x, p, c_{i}\right), i=1,2,3, \cdots$, is given by Equation (2.5).

Observe that the convergence of the series (2.5) depends upon the auxiliary constants $c_{i}, i=1,2,3, \cdots$. If the series (2.5) converges when $p=1$, one has

$$
h\left(x, 1, c_{i}\right)=u_{0}(x)+\sum_{j=1}^{\infty} N_{j}\left(x, u_{0}, u_{1}, \cdots, u_{j}\right) .
$$

The m-th order approximations are given by

$$
\tilde{u}\left(x, c_{1}, c_{2}, \cdots, c_{m}\right)=u_{0}(x)+\sum_{i=1}^{m} u_{i}\left(x, c_{1}, c_{2}, \cdots, c_{i}\right) .
$$

By substituting Equation (2.11) into Equation (2.1), we get the following expression for residual

$$
R\left(x, c_{1}, c_{2}, \cdots, c_{m}\right)=L\left(\tilde{u}\left(x, c_{1}, c_{2}, \cdots, c_{m}\right)\right)+g(x)+N\left(\tilde{u}\left(x, c_{1}, c_{2}, \cdots, c_{m}\right)\right) .
$$

If $R=0$, then $\tilde{u}$ will be the exact solution and this, in general, does not happen especially in nonlinear problems. In order to find the optimal values of $c_{i}, i=1,2,3, \cdots$, we apply the method of least squares as under

$$
J\left(c_{1}, c_{2}, \cdots, c_{m}\right)=\int_{a}^{b} R^{2}\left(x, c_{1}, c_{2}, \cdots, c_{m}\right) \mathrm{d} x,
$$

where $a$ and $b$ are numbers properly chosen in the domain of the problem. Next, minimizing $J$ with

$$
\frac{\partial J}{\partial c_{1}}=\frac{\partial J}{\partial c_{2}}=\cdots=\frac{\partial J}{\partial c_{m}}=0
$$

After knowing those constants, the approximate solution of order $m$ is well determined.

\section{Numerical Examples}

Example 1 Consider the second order initial value problem of Bratu type

$$
\left\{\begin{array}{l}
\frac{\mathrm{d}^{2}}{\mathrm{~d} x^{2}} u(x)=2 \mathrm{e}^{u(x)}, \\
u(0)=0, u^{\prime}(0)=0 .
\end{array}\right.
$$

The initial value problem (3.1) has $u(x)=-2 \ln \cos x$ as the exact solution.

Next, we apply the OHAM method to the initial value problem (3.1). We have

$g(x)=0, L(h(x, p))=h_{x x}(x, p)$ and $N(h(x, p))=-2 \mathrm{e}^{h(x, p)}$. Therefore, according to the OHAM method, we have

Problem of zero order: 


$$
\left\{\begin{array}{l}
\frac{\mathrm{d}^{2}}{\mathrm{~d} x^{2}} u_{0}(x)=0 \\
u_{0}(0)=0, u_{0}^{\prime}(0)=0
\end{array}\right.
$$

which has a solution $u_{0}(x)=0$.

Problem of first order:

$$
\left\{\begin{array}{l}
\frac{\mathrm{d}^{2}}{\mathrm{~d} x^{2}} u_{1}\left(x, c_{1}\right)=-2 c_{1} \\
u_{1}(0)=0, u_{1}^{\prime}(0)=0
\end{array}\right.
$$

Problem (3.3) has a solution

$$
u_{1}\left(x, c_{1}\right)=-c_{1} x^{2} .
$$

The problem of second order

$$
\left\{\begin{array}{l}
\frac{\mathrm{d}^{2}}{\mathrm{~d} x^{2}} u_{2}\left(x, c_{1}, c_{2}\right)=-2\left(c_{1}+c_{2}\right)-2 c_{1}^{2}+2 c_{1}^{2} x^{2} \\
u_{2}(0)=0, u_{2}^{\prime}(0)=0
\end{array}\right.
$$

The solution of Problem (3.5) is given by

Third order problem is

$$
u_{2}\left(x, c_{1}, c_{2}\right)=-\frac{1}{6}\left(6 c_{1} x^{2}+6 c_{2} x^{2}+6 c_{1}^{2} x^{2}-c_{1}^{2} x^{4}\right)
$$

$$
\left\{\begin{array}{l}
\frac{\mathrm{d}^{2}}{\mathrm{~d} x^{2}} u_{3}\left(x, c_{1}, c_{2}, c_{3}\right)=-2\left(c_{1}+c_{2}+c_{3}\right)-4\left(1-x^{2}\right)\left(c_{1} c_{2}+c_{1}^{2}\right)-\frac{2}{3}\left(3-6 x^{2}+2 x^{4}\right) c_{1}^{3}, \\
u_{3}(0)=0, u_{3}^{\prime}(0)=0
\end{array}\right.
$$

and its solution is given in the form

$$
u_{3}\left(x, c_{1}, c_{2}, c_{3}\right)=-x^{2}\left(c_{1}+c_{2}+c_{3}\right)-\frac{1}{3}\left(6 x^{2}-x^{4}\right)\left(c_{1} c_{2}+c_{1}^{2}\right)-\frac{1}{45}\left(45 x^{2}-15 x^{4}+2 x^{6}\right) c_{1}^{3} .
$$

Finally, fourth order problem is

$$
\begin{cases}\frac{\mathrm{d}^{2}}{\mathrm{~d} x^{2}} u_{4}\left(x, c_{1}, c_{2}, c_{3}, c_{4}\right)= & -2\left(c_{1}+c_{2}+c_{3}+c_{4}\right)-2\left(1-x^{2}\right)\left(4 c_{1} c_{2}+2 c_{1} c_{3}+3 c_{1}^{2}+c_{2}^{2}\right) \\ & -\left(6-12 x^{2}+4 x^{4}\right)\left(c_{1}^{2} c_{2}+c_{1}^{3}\right)-\frac{1}{45}\left(90-270 x^{2}+180 x^{4}-19 x^{6}\right) c_{1}^{4}, \\ u_{4}(0)=0, u_{4}^{\prime}(0)=0, & \end{cases}
$$

which has a solution in the form

$$
\begin{aligned}
u_{4}\left(x, c_{1}, c_{2}, c_{3}, c_{4}\right)= & -x^{2}\left(c_{1}+c_{2}+c_{3}+c_{4}\right)-\frac{1}{6}\left(6 x^{2}-x^{4}\right)\left(4 c_{1} c_{2}+2 c_{1} c_{3}+3 c_{1}^{2}+c_{2}^{2}\right) \\
& -\frac{1}{15}\left(45 x^{3}-15 x^{4}+2 x^{6}\right)\left(c_{1}^{2} c_{2}+c_{1}^{3}\right)-\frac{1}{2520}\left(2520 x^{2}-1260 x^{4}+336 x^{6}-19 x^{8}\right) c_{1}^{4} .
\end{aligned}
$$

Now, by using equations (3.4), (3.6), (3.8) and (3.10), the fourth order approximate solution, using OHAM with $p=1$, is given by

$$
\tilde{u}\left(x, c_{1}, c_{2}, c_{3}, c_{4}\right)=u_{0}(x)+u_{1}\left(x, c_{1}\right)+u_{2}\left(x, c_{1}, c_{2}\right)+u_{3}\left(x, c_{1}, c_{2}, c_{3}\right)+u_{4}\left(x, c_{1}, c_{2}, c_{3}, c_{4}\right) .
$$

Next, we follow the procedure presented in Section 2, we obtain the following values of $c_{i}$ 's:

$$
c_{1}=-0.9556156427, c_{2}=0.0942570476, c_{3}=0.0374885311 \text { and } c_{4}=-0.0116590295 \text { (Table 1). }
$$


Table 1. Absolute error between the exact solution and approximation solution.

\begin{tabular}{cccc}
\hline$x$ & Exact sol. & OHAM sol. & Error \\
\hline 0.0 & 0.00000000 & 0.00000000 & 0.00000000 \\
0.1 & 0.01001671 & 0.01001607 & $6.41021065 \times 10^{-7}$ \\
0.2 & 0.04026955 & 0.04025980 & $9.74693876 \times 10^{-6}$ \\
0.3 & 0.09138331 & 0.09133801 & $4.52998213 \times 10^{-5}$ \\
0.4 & 0.16445804 & 0.16433092 & $1.27118347 \times 10^{-4}$ \\
0.5 & 0.26116848 & 0.26089981 & $2.68671650 \times 10^{-4}$ \\
0.6 & 0.38393034 & 0.38344668 & $4.83656903 \times 10^{-4}$ \\
0.7 & 0.53617152 & 0.53533472 & $8.36799541 \times 10^{-4}$ \\
0.8 & 0.72278149 & 0.72118096 & $1.60053795 \times 10^{-3}$ \\
0.9 & 0.95088489 & 0.94723518 & $3.64970628 \times 10^{-3}$ \\
1.0 & 1.23125294 & 1.22186142 & $9.39151960 \times 10^{-3}$ \\
\hline
\end{tabular}

Example 2 In this example, let us consider the Bratu initial value problem

which has

$$
\frac{\mathrm{d}^{2}}{\mathrm{~d} x^{2}} u(x)=\pi^{2} \mathrm{e}^{u(x)}, \quad u(0)=0, u^{\prime}(0)=\pi,
$$

$$
u(x)=-\ln \left(1+\cos \frac{(2 x+1) \pi}{2}\right) \text { exact solution. }
$$

Now, we apply the OHAM method presented in previous section. In this example, we have

$$
g(x)=0, L(h(x, p))=h_{x x}(x, p) \text { and } N(h(x, p))=-\pi^{2} \mathrm{e}^{h(x, p)} . \text { Now, }
$$

Problem of zero order:

$$
\left\{\begin{array}{l}
\frac{\mathrm{d}^{2}}{\mathrm{~d} x^{2}} u_{0}(x)=0 \\
u_{0}(0)=0, u_{0}^{\prime}(0)=\pi
\end{array}\right.
$$

Problem (3.13) has a solution $u_{0}(x)=\pi x$.

Problem of first order:

$$
\left\{\begin{array}{l}
\frac{\mathrm{d}^{2}}{\mathrm{~d} x^{2}} u_{1}(x)=-\pi^{2} c_{1}\left(1+\pi x+\frac{\pi^{2}}{2} x^{2}\right), \\
u_{1}(0)=0, u_{1}^{\prime}(0)=0 .
\end{array}\right.
$$

The solution of Problem (3.14) is given by

$$
u_{1}(x)=-\frac{1}{24} \pi^{2} x^{2} c_{1}\left(12+4 \pi x+\pi^{2} x^{2}\right) .
$$

The problem of second order

$$
\left\{\begin{array}{l}
\frac{\mathrm{d}^{2}}{\mathrm{~d} x^{2}} u_{2}\left(x, c_{1}, c_{2}\right) \\
=-\frac{\pi^{2}}{2}\left(2+2 \pi x+\pi^{2} x^{2}\right)\left(c_{1}+c_{2}\right) \\
-\frac{\pi^{2}}{24}\left(24+24 \pi x-16 \pi^{3} x^{3}-5 \pi^{4} x^{4}-\pi^{5} x^{5}\right) c_{1}^{2} \\
u_{2}(0)=0, u_{2}^{\prime}(0)=0
\end{array}\right.
$$


and its solution is given by

$$
\begin{aligned}
u_{2}(x)= & -\frac{\pi^{2} x^{2}}{24}\left(12+4 \pi x+\pi^{2} x^{2}\right)\left(c_{1}+c_{2}\right) \\
& -\frac{\pi^{2} x^{2}}{5040}\left(2520+840 \pi x-168 \pi^{3} x^{3}-35 \pi^{4} x^{4}-5 \pi^{5} x^{5}\right) c_{1}^{2}
\end{aligned}
$$

Third order problem is

$$
\left\{\begin{aligned}
& \frac{\mathrm{d}^{2}}{\mathrm{~d} x^{2}} u_{3}\left(x, c_{1}, c_{2}, c_{3}\right) \\
&=-\frac{\pi^{2}}{2}\left(2+2 \pi x+\pi^{2} x^{2}\right)\left(c_{1}+c_{2}+c_{3}\right) \\
&-\frac{\pi^{2}}{12}\left(24+24 \pi x-16 \pi^{3} x^{3}-5 \pi^{4} x^{4}-\pi^{5} x^{5}\right)\left(c_{1} c_{2}+c_{1}^{2}\right) \\
&-\pi^{2}\left(1+\pi x-\frac{1}{2} \pi^{2} x^{2}-\frac{4}{3} \pi^{3} x^{3}-\frac{1}{4} \pi^{4} x^{4}+\frac{3}{40} \pi^{5} x^{5}+\frac{3}{40} \pi^{6} x^{6}+\frac{5}{336} \pi^{7} x^{7}+\frac{5}{2688} \pi^{8} x^{8}\right) c_{1}^{3}, \\
& u_{3}(0)=0, u_{3}^{\prime}(0)=0 .
\end{aligned}\right.
$$

The solution of Problem (3.18) is given by

$$
\begin{aligned}
u_{3}\left(x, c_{1}, c_{2}, c_{3}\right) \\
=-\frac{\pi^{2} x^{2}}{24}\left(12+4 \pi x+\pi^{2} x^{2}\right)\left(c_{1}+c_{2}+c_{3}\right) \\
-\frac{\pi^{2}}{2520}\left(2520 x^{2}+840 \pi x^{3}-168 \pi^{3} x^{5}-35 \pi^{4} x^{6}-5 \pi^{5} x^{7}\right)\left(c_{1} c_{2}+c_{1}^{2}\right) \\
-\pi^{2}\left(\frac{x^{2}}{2}+\frac{\pi x^{3}}{6}-\frac{\pi^{2} x^{4}}{24}-\frac{\pi^{3} x^{5}}{15}-\frac{\pi^{4} x^{6}}{120}+\frac{\pi^{5} x^{7}}{560}+\frac{3 \pi^{6} x^{8}}{2240}+\frac{5 \pi^{7} x^{9}}{24192}+\frac{\pi^{8} x^{10}}{48384}\right) c_{1}^{3} .
\end{aligned}
$$

In the end, the fourth order problem is given by

$$
\left\{\begin{aligned}
\frac{\mathrm{d}^{2}}{\mathrm{~d} x^{2}} u_{4}\left(x, c_{1}, c_{2}, c_{3}, c_{4}\right) \\
=-\frac{\pi^{2}}{2}\left(2+2 \pi x+\pi^{2} x^{2}\right)\left(c_{1}+c_{2}+c_{3}+c_{4}\right) \\
\quad-\frac{\pi^{2}}{24}\left(24+24 \pi x-16 \pi^{3} x^{3}-5 \pi^{4} x^{4}-\pi^{5} x^{5}\right)\left(4 c_{1} c_{2}+2 c_{1} c_{3}+3 c_{1}^{2}+c_{2}^{2}\right) \\
\quad-\frac{\pi^{2}}{8960}\left(26880+26880 \pi x-13440 \pi^{2} x^{2}-35840 \pi^{3} x^{3}-6720 \pi^{4} x^{4}\right. \\
\left.+2016 \pi^{5} x^{5}+2016 \pi^{6} x^{6}+400 \pi^{7} x^{7}+50 \pi^{8} x^{8}\right)\left(c_{1}^{2} c_{2}+c_{1}^{3}\right) \\
\quad-\frac{\pi^{2}}{161280}\left(161280+161280 \pi x-161280 \pi^{2} x^{2}-322560 \pi^{3} x^{3}-20160 \pi^{4} x^{4}+56448 \pi^{5} x^{5}\right. \\
\left.+32032 \pi^{6} x^{6}+1888 \pi^{7} x^{7}-1660 \pi^{8} x^{8}-740 \pi^{9} x^{9}-110 \pi^{10} x^{10}-10 \pi^{11} x^{11}\right) c_{1}^{4}, \\
u_{4}(0)=0, u_{4}^{\prime}(0)=0 .
\end{aligned}\right.
$$

which has a solution in the form 
Table 2. Absolute error between the exact solution and approximation solution.

\begin{tabular}{cccc}
\hline$x$ & Exact sol. & OHAM sol. & Error \\
\hline-0.3 & -0.59278360 & -0.59050589 & $2.27771434 \times 10^{-3}$ \\
-0.2 & -0.46234012 & -0.46195092 & $3.89206013 \times 10^{-4}$ \\
-0.1 & -0.26927647 & -0.26926585 & $1.06182822 \times 10^{-5}$ \\
0.0 & 0.00000000 & 0.00000000 & 0.00000000 \\
0.1 & 0.36964005 & 0.36959323 & $4.68162342 \times 10^{-5}$ \\
0.2 & 0.88621083 & 0.88427218 & $1.93865546 \times 10^{-3}$ \\
0.3 & 1.65557083 & 1.63022895 & $2.53418803 \times 10^{-2}$ \\
\hline
\end{tabular}

$$
\begin{aligned}
u_{4}\left(x, c_{1}, c_{2}, c_{3}, c_{4}\right) \\
=-\frac{\pi^{2} x^{2}}{24}\left(12+4 \pi x+\pi^{2} x^{2}\right)\left(c_{1}+c_{2}+c_{3}+c_{4}\right)-\frac{\pi^{2} x^{2}}{2520}\left(2520+840 \pi x-168 \pi^{3} x^{3}-35 \pi^{4} x^{4}-5 \pi^{5} x^{5}\right) \\
\quad \cdot\left(2 c_{1} c_{2}+c_{1} c_{3}\right)-\frac{\pi^{2} x^{2}}{5040}\left(2520+840 \pi x-168 \pi^{3} x^{3}-35 \pi^{4} x^{4}-5 \pi^{5} x^{5}\right)\left(3 c_{1}^{2}+c_{2}^{2}\right) \\
\quad-\frac{\pi^{2} x^{2}}{8960}\left(13440+4480 \pi x-1120 \pi^{2} x^{2}-1792 \pi^{3} x^{3}-224 \pi^{4} x^{4}+48 \pi^{5} x^{5}+36 \pi^{6} x^{6}+\frac{50}{9} \pi^{7} x^{7}+\frac{5}{9} \pi^{8} x^{8}\right) \\
\quad\left(c_{1}^{2} c_{2}+c_{1}^{3}\right)-\frac{\pi^{2} x^{2}}{161280}\left(80640+26880 \pi x-13440 \pi^{2} x^{2}-16128 \pi^{3} x^{3}-672 \pi^{4} x^{4}+1344 \pi^{5} x^{5}+572 \pi^{6} x^{6}\right. \\
\left.+\frac{236}{9} \pi^{7} x^{7}-\frac{166}{9} \pi^{8} x^{8}-\frac{74}{11} \pi^{9} x^{9}-\frac{5}{6} \pi^{10} x^{10}-\frac{5}{78} \pi^{11} x^{11}\right) c_{1}^{4} .
\end{aligned}
$$

Now, by using Equations (3.4), (3.6), (3.8) and (3.10), the fourth order approximate solution, using OHAM with $p=1$, is given by

$$
\tilde{u}\left(x, c_{1}, c_{2}, c_{3}, c_{4}\right)=u_{0}(x)+u_{1}\left(x, c_{1}\right)+u_{2}\left(x, c_{1}, c_{2}\right)+u_{3}\left(x, c_{1}, c_{2}, c_{3}\right)+u_{4}\left(x, c_{1}, c_{2}, c_{3}, c_{4}\right) .
$$

Next, we follow the procedure presented in Section 0.2, we obtain the following values of $c_{i}$ 's: $c_{1}=-1.0391835661, \quad c_{2}=-0.0042471858, c_{3}=0.0000013808$ and $c_{4}=0.0001595594$ (Table 2).

\section{Final Remarks}

Throughout this paper, an technique for obtaining a numerical solution for second order initial value problems of Bratu-type, is optimal homotopy asymptotic method (OHAM). The main advantage of the used technique is achieving high accurate approximate solutions. In the numerical tables and graphics, our numerical results are compared with the exact ones.

\section{References}

[1] Herişanu, N., Marinca, V., Dordea T. and Madescu, G. (2008) A New Analytical Approach to Nonlinear Vibration of an Electrical Machine. Proceedings of the Romanian Academy, Series A, 9, 229-236.

[2] Ali, J., Islam, S., Islam, S. and Zamand, G. (2010) The Solution of Multipoint Boundary Value Problems by the Optimal Homotopy Asymptotic Method. Computers \& Mathematics with Applications, 59, 2000-2006. http://dx.doi.org/10.1016/j.camwa.2009.12.002

[3] Ene, R.D., Marinca, V., Negrea, R. and Caruntu, B. (2012) Optimal Homotopy Asymptotic Method for Solving a Nonlinear Problem in Elasticity. Symbolic and Numeric Algorithms for Scientific Computing (SYNASC), 14th International Symposium, Timisoara, 26-29 September 2012, 98-102.

[4] Esmaeilpour, M. and Ganji, D.D. (2010) Solution of the Jeffery-Hamel Flow Problem by Optimal Homotopy Asymptotic Method. Computers \& Mathematics with Applications, 59, 3405-3411. http://dx.doi.org/10.1016/j.camwa.2010.03.024

[5] Hashmi, M.S., Khan, N. and Iqbal, S. (2012) Optimal Homotopy Asymptotic Method for Solving Nonlinear Fredholm Integral Equations of Second Kind. Applied Mathematics and Computation, 218, 10982-10989. 
http://dx.doi.org/10.1016/j.amc.2012.04.059

[6] Marinca, V., Herişanu, N., Bota, C. and Marinca, B. (2009) An Optimal Homotopy Asymptotic Method Applied to the Steady Flow of a Fourth-Grade Fluid Past a Porous Plate. Applied Mathematics Letters, 22, 245-251. http://dx.doi.org/10.1016/j.aml.2008.03.019

[7] Abukhaled, M., Khuri, S. and Sayfy, A. (2012) Spline-Based Numerical Treatments of Bratu-Type Equations. Palestine Journal of Mathematics, 1, 63-70.

[8] Wazwaz, A. (2012) A Reliable Study for Extensions of the Bratu Problem with Boundary Conditions. Mathematical Methods in the Applied Sciences, 35, 845-856. http://dx.doi.org/10.1002/mma.1616

[9] Batiha, B. (2010) Numerical Solution of Bratu-Type Equations by the Variational Iteration Method. Hacettepe Journal of Mathematics and Statistics, 39, 23-29.

[10] Feng, X., He, Y. and Meng, J. (2008) Application of Homotopy Perturbation Method to the Bratu-Type Equations. Topological Methods in Nonlinear Analysis, 31, 243-252.

[11] Rashidinia, J., Maleknejad, K. and Taheri, N. (2013) Sinc-Galerkin Method for Numerical Solution of the Bratu's Problems. Numerical Algorithms, 62, 1-11. http://dx.doi.org/10.1007/s11075-012-9560-3

[12] Syam, M.I. and Hamdan, A. (2006) An Efficient Method for Solving Bratu Equations. Applied Mathematics and Computation, 176, 704-713. http://dx.doi.org/10.1016/j.amc.2005.10.021

[13] Wazwaz, A. (2005) Adomian Decomposition Method for a Reliable Treatment of the Bratu-Type Equations. Applied Mathematics and Computation, 166, 652-663. http://dx.doi.org/10.1016/j.amc.2004.06.059 